\title{
MicroRNA-196b targets COSMC in pediatric IgA nephropathy
}

\author{
QIANG SUN ${ }^{1}$, JINGCHAO LAN $^{1}$, HONG ZHANG $^{2}$, NAN ZHOU $^{1}$, YING LIANG $^{1}$ and XIAORONG LIU ${ }^{1}$ \\ ${ }^{1}$ Department of Nephrology, Beijing Children's Hospital, Capital Medical University, National Center for Children's Health, \\ Beijing Key Laboratory of Pediatric Chronic Kidney Disease and Blood Purification, Key Laboratory of \\ Major Diseases in Children, Ministry of Education, Beijing 100045; ${ }^{2}$ Pediatric Department, Shunyi Women's \\ and Children's Hospital of Beijing Children's Hospital, Beijing 101300, P.R. China
}

Received July 5, 2019; Accepted November 28, 2019

DOI: $10.3892 / \mathrm{mmr} .2020 .11015$

\begin{abstract}
The aim of the present study was to investigate the effect of microRNAs (miRNAs) on the expression level of core $1 \beta$ 3-galactosyltransferase-specific molecular chaperone (COSMC) in immunoglobulin A nephropathy (IgAN). miRNA expression levels were determined in pediatric patients with $\operatorname{IgAN}$ (IgAN group), in patients with other renal diseases (control group) and healthy pediatrics (control group). The target miRNAs of COSMC were investigated in the present study. Western blot analysis was performed to examine the effects of miRNAs on COSMC expression levels. In addition, galactose-deficient IgA1 (Gd-IgA1) expression levels were detected following the addition of miRNA-196b. The present results suggested that the expression levels of 205 miRNAs significantly differed between the IgAN and healthy control groups. The present results also suggested that miRNA-196b and miRNA-33a-3p targeted COSMC, and that miRNA-196b expression in B lymphocytes was significantly higher in the IgAN group compared with the control group $(\mathrm{P}<0.0001)$. However, COSMC expression level was significantly downregulated in isolated B lymphocytes transfected with miRNA-196b mimics, but Gd-IgA1 expression levels were increased. Therefore, miRNA-196b may play a role in the formation of Gd-IgA1 and $\operatorname{IgAN}$ pathogenesis via COSMC regulation.
\end{abstract}

Correspondence to: Professor Xiaorong Liu, Department of Nephrology, Beijing Children's Hospital, Capital Medical University, National Center for Children's Health, Beijing Key Laboratory of Pediatric Chronic Kidney Disease and Blood Purification, Key Laboratory of Major Diseases in Children, Ministry of Education, 56 Nanlishi Road, Beijing 100045, P.R. China

E-mail: desin2000@sina.com

Abbreviations: miRNA, microRNA; IgAN, immunoglobulin A nephropathy; C1 $\beta 3 \mathrm{Gal}-\mathrm{T}$, core1 $\beta 3$-galactosyltransferase; COSMC, C1ß3Gal-T-specific molecular chaperone

Key words: IgA, pediatrics, core1 $\beta 3$-galactosyltransferase-specific molecular chaperone, microRNA-196

\section{Introduction}

Immunoglobulin A ( $\operatorname{Ig} A)$ nephropathy $(\operatorname{IgAN})$, one of the most common causes of primary glomerulonephritis in pediatrics, was conventionally considered a benign disease (1). However, $\operatorname{Ig} \mathrm{AN}$ is now known to be a progressive renal disease, with $20-30 \%$ of patients developing end-stage renal failure over 20-30 years $(2,3)$.

Although the pathogenesis of IgAN remains unknown, it has been reported that aberrant glycosylation and glomerular mesangial deposition of IgA1 serve critical roles in its pathogenesis (4-6). Deficiency of core $1 \beta 3$-galactosyltransferase (C1 $\beta 3$ Gal-T) is associated with aberrant glycosylation of IgA1 (7). Moreover, Ju and Cummings (8) demonstrated that the unique molecular chaperone of $\mathrm{C} 1 \beta 3 \mathrm{Gal}-\mathrm{T}, \mathrm{C} 1 \beta 3 \mathrm{Gal}-\mathrm{T}$-specific molecular chaperone (C1GALT1C1/COSMC) is vital for the activity of $\mathrm{C} 1 \beta 3 \mathrm{Gal}-\mathrm{T}$ (8-10). Previous studies have reported that COSMC expression levels in B lymphocytes were lower in patients with IgAN compared with healthy controls and patients with other renal diseases $(9,11)$. However, the mechanisms underlying COSMC expression are not fully understood (10). Serino et al $(12,13)$ indicated that microRNA (miRNA)-148b targeted and regulated $\mathrm{C} 1 \beta 3 \mathrm{Gal}-\mathrm{T}$ expression. Moreover, as a molecular chaperone, the regulation of COSMC is crucial for its biological functions, but the mechanisms remain largely unknown (10). Therefore, the present study aimed to investigate the effect of miRNAs on COSMC expression.

\section{Materials and methods}

Screening for miRNA expression levels in pediatric patients with IgAN and healthy controls. The study cohort included 9 pediatric patients with $\operatorname{Ig} \mathrm{AN}$ (IgAN group), who were independently diagnosed by two experienced pathologists, and 6 healthy pediatrics (control group) that were recruited by Beijing Children's Hospital between January 2015 and December 2018. There were no significant differences between the two groups in terms of age and sex $(\mathrm{P}>0.05$; Table I). Peripheral blood samples $(5 \mathrm{ml})$ were from the veins of the patients and healthy controls. Plasma was obtained by centrifugation at $1,710 \mathrm{x}$ g at $4^{\circ} \mathrm{C}$ for $5 \mathrm{~min}$. Samples were subsequently stored at $-80^{\circ} \mathrm{C}$ until required for subsequent analysis. B lymphocytes were isolated, and miRNAs were extracted for single-end second-generation sequencing. 
Briefly, total RNA was extracted from the plasma using TRIzol $^{\circledR}$ reagent (Invitrogen; Thermo Fisher Scientific, Inc.) and a miRNeasy Mini kit (Qiagen, Inc.). Hybridization and ligation were performed for small RNA fractions of $<50$ nucleotide using a NEBNext ${ }^{\circledR}$ Multiplex Oligos for Illumina ${ }^{\circledR}$ (Methylated Adaptor, Index Primers Set 1) kit (cat. no. E7535L; New England Biolabs, Inc.). The miRNAs were subsequently sequenced using the Illumina HiSeq 2000 Sequencing system. All procedures performed involving human participants were approved by the Medical Ethics Committee of Beijing Children's Hospital, Capital Medical University (IRB approval no. 2014-12). Informed consent was obtained from the parents of all participants prior to the study.

Lymphocyte isolation. EDTA-anticoagulated blood samples $(5 \mathrm{ml})$ from the IgAN group were collected the day after kidney biopsy and immediately sent for cell isolation and processing. Fresh blood samples $(2 \mathrm{ml})$ were mixed with Hanks' balanced salt solution $(2 \mathrm{ml})$ at room temperature for $30 \mathrm{~min}$. Subsequently, AstroMACS Separation Buffer (Miltenyi Biotec, Inc.) was added to the liquid surface and centrifuged at room temperature at 3,000 rcf for $20 \mathrm{~min}$. The second layer of cells was then collected and mixed with $5 \mathrm{ml}$ Hanks' balanced salt solution, and the mixture was centrifuged twice at room temperature and 2,000 $\mathrm{rcf}$ for $15 \mathrm{~min}$ to separate the lymphocytes. Then, $1 \mathrm{ml}$ Dynabeads (Thermo Fisher Scientific, Inc.) was added to $1 \mathrm{ml}$ PBS and mixed by shaking. The tube containing the suspension was placed in a magnetic system at room temperature for $1 \mathrm{~min}$. The supernatant was collected, and the process was repeated 2-3 times. The separated lymphocytes were resuspended and cell counts were determined to adjust the cell concentration to $1 \times 10^{5} / \mathrm{ml}$. The suspension was shaken for $25 \mathrm{~min}$ at $4^{\circ} \mathrm{C}$ before placing the tube in the magnetic system for $2 \mathrm{~min}$. The supernatant was removed, $1 \mathrm{ml}$ PBS placed in the magnetic system for $1 \mathrm{~min}$ was added and the supernatant was collected; this procedure was repeated four times. To purify $\mathrm{CD} 19^{+}$cells, the magnetic bead suspension was resuspended and $10 \mu \mathrm{l}$ DETACHaBEAD reagent (Thermo Fisher Scientific, Inc.) was added per $25 \mu 1$ magnetic bead suspension and then mixed. Subsequently, after washing with $1 \mathrm{ml} \mathrm{10 \%} \mathrm{PBS,} \mathrm{the} \mathrm{supernatant} \mathrm{was} \mathrm{collected} \mathrm{by} \mathrm{centrifuga-}$ tion at room temperature and $600 \mathrm{rcf}$ for $6 \mathrm{~min}$ to remove the residual DETACHaBEAD reagent. The cells were then resuspended for subsequent experiments.

miRNA target prediction. The bioinformatics software PicTar (http://www.pictar.org/) and TargetScan version 7.1 (http://www.targetscan.org) were used to analyze the corresponding miRNAs of COSMC.

Validation tests of the IgAN and control groups. To expand the sample size, a further 15 pediatric patients with $\operatorname{IgAN}$ and 15 controls (four healthy controls and 11 pediatric patients with other renal diseases) were recruited between January 2013 to December 2018 from Beijing Children's Hospital. No significant differences were observed among these three groups in terms of age, sex and $24 \mathrm{~h}$ urine total protein results, except the urine proteins in the healthy control group (Table II). B lymphocytes
Table I. Clinical data of the IgAN and healthy control groups.

\begin{tabular}{lccc}
\hline Variables & $\begin{array}{c}\text { IgAN } \\
\text { group }\end{array}$ & $\begin{array}{c}\text { Healthy } \\
\text { control group }\end{array}$ & P-value \\
\hline Cases & 9 & 6 & \\
Age (years) & $9.03 \pm 2.99$ & $9.68 \pm 2.57$ & 0.607 \\
Sex & & & 0.659 \\
Male & 7 & 5 & \\
Female & 2 & 1 & \\
\hline
\end{tabular}

There were no significant differences between the IgAN and healthy control groups in terms of the baseline levels of age and sex before the experiments. IgAN, immunoglobulin A nephropathy.

were isolated from $5 \mathrm{ml}$ peripheral blood samples with $5 \mathrm{ml}$ EDTA as an anticoagulant. Total RNA was extracted using an miRNeasy kit (cat. no. 217184; Qiagen $\mathrm{GmbH}$ ) according to the manufacturer's instructions. A spectrophotometer was used to detect the absorbance at $450 \mathrm{~nm}$ of the extracted RNA for analysis. RNA was reverse transcribed into cDNA using the PrimeScript RT Reagent kit (Takara Biotechnology Co., Ltd.) at $37^{\circ} \mathrm{C}$ for $1 \mathrm{~h}$. Expression levels were detected by qPCR using the miScript SYBR ${ }^{\circledR}$ Green PCR kit (Qiagen, Inc.), according to the manufacturer's protocol. The following primers were used for the qPCR: MiRNA-196b-5p forward, 5'-CTCAACTGGTGT CGTGGAGTCGGCAATTCAGTTGAGCCCAACAA-3' and reverse, 5'-ACACTCCAGCTGGGTAGGTAGTTTCCTGTT-3'; and RNU6 forward, 5'-CTCATGACCACAGTCCATGCC-3' and reverse, 5'-GGCATGGACTGTGGTCATGAG-3'. The thermocycling conditions for the qPCR were as follows: Initial denaturation at $95^{\circ} \mathrm{C}$ for $30 \mathrm{sec}$; and 40 cycles of $95^{\circ} \mathrm{C}$ for $5 \mathrm{sec}$ and $60^{\circ} \mathrm{C}$ for $30 \mathrm{sec}$. The relative miRNA-196b expression levels were calculated using the $2^{-\Delta \Delta \mathrm{Cq}}$ method (14).

Western blotting analysis of effect of miRNA-196b on the expression of COSMC. Isolated B lymphocytes of pediatric patients with IgAN from the present study were divided into three groups: i) Control group (untreated); ii) miRNA-196b group which contained the miRNA-196b-transfected B lymphocytes; and iii) the negative control (NC) group, which contained the NC-transfected B lymphocytes. Total cellular proteins were extracted from $1 \times 10^{6} \mathrm{~B}$ lymphocytes $/ \mathrm{ml}$ using RIPA lysis buffer [50 mM Tris (pH 7.4), $150 \mathrm{mM} \mathrm{NaCl}$, $1 \%$ Triton $\mathrm{X}-100,1 \%$ sodium deoxycholate and $0.1 \%$ SDS; Sigma-Aldrich; Merck KGaA] and quantified using the bicinchoninic acid protein assay. The total proteins $(20 \mu \mathrm{g} / \mathrm{lane})$ obtained from the cells were separated using 12\% SDS-PAGE and then transferred to PVDF membranes $(0.45 \mu \mathrm{m}$; Roche Diagnostics). After blocking with $5 \%$ non-fat dry milk at $4^{\circ} \mathrm{C}$ overnight, the membranes were incubated with antibodies against anti-COSMC (1:2,000; cat. no. 68310; Abcam) or $\beta$-actin (1:3,000; cat. no. 4970; Cell Signaling Technology, Inc.) at $4^{\circ} \mathrm{C}$ overnight. Then, the membranes were incubated at room temperature for $1 \mathrm{~h}$ with secondary antibodies labeled with horseradish peroxidase (HRP)-conjugated goat anti-rabbit IgG-HRP (cat. no. sc-2005; 1:5,000; Santa Cruz Biotechnology, Inc.) or goat anti-mouse IgG-HRP 
Table II. Clinical data of the IgAN and control groups.

\begin{tabular}{lcccr}
\hline Variables & IgAN group & Healthy control group & Other renal disease group & P-value \\
\hline Cases & 15 & 4 & 11 & 0.470 \\
Average age (years) & $9.60 \pm 2.55^{\mathrm{a}}$ & $8.68 \pm 2.80$ & $8.80 \pm 2.92$ & 0.907 \\
Sex & 12 & 3 & 8 & 0.328 \\
Male & 3 & 1 & 3 & $751 \pm 511$ \\
Female & $819.4 \pm 233.0^{\mathrm{b}}$ & & \\
$24 \mathrm{~h}$ urine total protein $(\mathrm{mg})$ & & & \\
a'Mean $\pm \mathrm{SD}$; ${ }^{\mathrm{b}}$ mean \pm SEM. There were no significant differences among the three groups in terms of the baseline levels of age, sex and $24 \mathrm{~h}$ \\
urine total protein before the experiments. IgAN, immunoglobulin A nephropathy. \\
\hline
\end{tabular}

(cat. no. sc-2004; 1:5,000; Santa Cruz Biotechnology, Inc.). An enhanced chemiluminescence kit (Amersham; GE Healthcare Life Sciences) was used to visualize the protein bands.

Comparative analysis after miRNA-196b transfection. In our previous study (11), three groups of patients were included: i) 26 biopsy-proven pediatric patients with $\operatorname{Ig} \mathrm{AN}$; ii) 13 healthy pediatrics (control group); and iii) 11 pediatric patients with other renal diseases, including hereditary nephritis and membranous nephropathy (second control group). In the present study, B lymphocytes of pediatric patients with IgAN from the above cohort (26 biopsy-proven pediatric patients with IgAN) were transfected with miRNA-196b mimics (Thermo Fisher Scientific, Inc.; forward, 5'-CTCAACTGG TGTCGTGGAGTCGGCAATTCAGTTGAGCCCAACAA-3' and reverse, 5'-ACACTCCAGCTGGGTAGGTAGTTTCCTG TT-3') or its negative control (NC; Thermo Fisher Scientific, Inc.; forward, 5'-CTCATGACCACAGTCCATGCC-3' and reverse, 5'-GGCATGGACTGTGGTCATGAG-3'). A total of $20 \mu \mathrm{g} / \mathrm{ml}$ miRNA-196b or the NC was transfected into $1 \times 10^{6} \mathrm{~B}$ lymphocytes/ml in the IgAN group using Lipofectamine ${ }^{\circledR} 3000$ reagent (Invitrogen; Thermo Fisher Scientific, Inc.), according to the manufacturer's protocol, for $48 \mathrm{~h}$ prior to subsequent experimentation. Then, Gd-IgA1 expression levels were determined using a Gd-IgA1 ELISA kit (cat. no. F10079; Shanghai Fanke Industry Co., Ltd.), according to the manufacturer's protocol. Informed consent was provided by the patients for the use of their samples in this study.

Correlations between miRNA-196b expression levels and clinical data of patients. In addition to the detection of miRNA-196b expression levels, the clinical data of the IgAN group $(n=14 ; 1$ patient was excluded due to in insufficient clinical data) was recorded and correlation analysis was performed using Pearson's correlation coefficient. Renal function was determined by detecting the levels of serum blood urea nitrogen (BUN) using UREA reagent (cat. no. OSR6134; Beckman Coulter, Inc.), according to the manufacturer's protocol, the serum creatinine $(\mathrm{sCr})$ levels using a Creatinine Test kit [cat. no. MC1212B; Merit Choice Bioengineering (Beijing) Co., Ltd.], according to the manufacturer's protocol, and the glomerular filtration rate (GFR) using the Schwartz formula, as previously described (15). The $24 \mathrm{~h}$ urine total protein concentration was determined using Total protein UC FS reagent (Diasys Diagnostic Systems $\mathrm{GmbH}$ ), according to the manufacturer's protocol, and urine red blood cells were collected from urine by centrifugation $\left(500 \mathrm{x} \mathrm{g} ; 5 \mathrm{~min} ; 20-25^{\circ} \mathrm{C}\right)$ and counted using a phase contrast microscope (magnification, $\mathrm{x} 400$ ).

CD3, CD4 and CD8 expression levels in peripheral blood $\mathrm{T}$ cells were assessed using flow cytometric analysis. Briefly, $2 \mathrm{ml}$ EDTA anti-coagulation peripheral blood was collected from the patients with IgAN group and $100 \mu 1$ whole blood from the sample was added to each tube. Samples were blocked with 3\% FBS (cat. no. 04-001-1; Biological industries) at room temperature for $10 \mathrm{~min}$ and then, $5 \mu \mathrm{l} \mathrm{C}-\mathrm{S}^{\circledR}$ tetraCHROMETM $^{\text {TM } 45-F I T C / C D 4-R D 1 / C D 8-E C D / C D 3-P C 5 ~}$ antibody (cat. no. 6607013; Beckman Coulter, Inc.) was added and incubated in the dark for $10 \mathrm{~min}$ at room temperature. Subsequently, $500 \mu 1$ hemolysin was added/tube, mixed by shaking and incubated at room temperature for $10 \mathrm{~min}$ in the dark, followed by incubation at room temperature for $10 \mathrm{~min}$ in the dark of $500 \mu \mathrm{l}$ PBS. T cells were visualized using a Beckman Coulter Cytomics FC-500 Flow Cytometer (Beckman Coulter, Inc.) and data was analyzed using CXP analysis software (version 2.0; Beckman Coulter, Inc.).

Histochemistry. Renal histological manifestations were observed by histochemical staining and graded according to Lee's glomerular grading system (16). Briefly, renal tissues from the right kidney of the 14 patients mentioned above were collected following renal biopsy and fixed in $10 \%$ formalin for $4 \mathrm{~h}$ at room temperature. Tissues were embedded in paraffin and cut into 3- $\mu \mathrm{m}$ thick sections. Sections were deparaffinized with xylene, rehydrated in a descending series of ethanal and routinely stained hematoxylin and eosin, periodic acid-Schiff (PAS), PAS + methenamine, and Masson's trichrome. Stained sections were observed using a light microscope (magnification, $x 40, x 100, x 200$ or $x 400)$ to determine patient renal pathology. Based on the results, the patients were divided into two groups: IgAN with grade I-II renal damage as group A and IgAN with grade III-V renal damage as group B.

Statistical analysis. Data are presented as the mean \pm SD of $\geq 2$ averaged replicates of independent experiments. Quantitative data were compared using one-way ANOVA followed by Least Significant Difference post hoc test (Table II) or Tukey's test (Fig. 1), or Student's t-test (Table III). Crosstabs and $\mathrm{c}^{2}$ tests 
Table III. Correlation between miRNA-196b expression levels and clinical data.

\begin{tabular}{|c|c|c|c|c|c|}
\hline Variables in IgAN group & Mean & $\mathrm{SD}$ or $\mathrm{SEM}^{\mathrm{c}}$ & $r$ & $\mathrm{n}$ & P-value \\
\hline $24 \mathrm{~h}$ urine total protein, $\mathrm{mg}$ & 821.47 & $235.6^{\mathrm{c}}$ & -0.590 & & $0.021^{\mathrm{a}}$ \\
\hline CD3 & 70.83 & 7.47 & 0.361 & & 0.186 \\
\hline CD4 & 29.51 & 6.35 & -0.044 & & 0.875 \\
\hline CD8 & 33.55 & 5.06 & 0.066 & & 0.814 \\
\hline BUN, mmol/l & 4.79 & 2.24 & -0.340 & & 0.215 \\
\hline $\mathrm{sCr}, \mu \mathrm{mol} / 1$ & 52.65 & 16.97 & -0.422 & & 0.117 \\
\hline $\mathrm{GFR}, \mathrm{ml} / \mathrm{min} / 1.73 \mathrm{~m}^{2}$ & 103.14 & 20.97 & 0.317 & & 0.250 \\
\hline $\mathrm{RBC}, / \mathrm{HP}$ & 80.8 & 22.61 & 0.478 & & 0.071 \\
\hline Pathology ${ }^{\mathrm{b}}$ & & & & & 0.609 \\
\hline Grade I-II & & & & 4 & \\
\hline Grade III-V & & & & 10 & \\
\hline
\end{tabular}

${ }^{\mathrm{a}} \mathrm{P}<0.05$, ${ }^{\mathrm{b}}$ using Lee's glomerular grading system (16), ${ }^{\mathrm{c}} \mathrm{SEM}$. The clinical data the IgAN group were recorded and analyzed for correlations between miRNA-196b expression levels and clinical data. There were no correlations between miRNA-196b expression levels and CD3, CD4, CD8, BUN, sCr and GFR ( $\mathrm{P}=0.186,0.875,0.814,0.215,0.117$ and 0.250 , respectively). No significant differences were identified between the two different renal pathological groups and the miRNA-196b expression levels (Student's t-test; $\mathrm{P}=0.609$ ). However, there was a significant correlation between miRNA-196b expression levels and the $24 \mathrm{~h}$ urine total protein results $(\mathrm{P}=0.021)$. BUN, blood urea nitrogen; $\mathrm{sCr}$, serum creatinine; GFR, glomerular filtration rate; RBC, red blood cell; HP, high power; IgAN, immunoglobulin A nephropathy.

were used to compare frequencies. Pearson's correlation coefficient was used to identify correlations among factors. All data analyses were performed using the SPSS software (version 18.0; SPSS, Inc.). $\mathrm{P}<0.05$ was considered to indicate a statistically significant difference.

\section{Results}

miRNA expression levels in pediatric patients with IgAN and healthy controls. B lymphocytes were isolated from the peripheral blood samples of 9 pediatric patients with IgAN and 6 healthy controls. miRNAs extracted from these cells were subjected to second-generation sequencing. Bioinformatics analysis detected 205 miRNAs with significant differences in expression levels between the $\operatorname{Ig} \mathrm{AN}$ and control groups $\left(\mathrm{P}<0.05 ; \mathrm{P}=0.013-1.41 \times 10^{-22}\right.$; data not shown $)$.

miRNA target prediction. The target miRNAs of COSMC were investigated and predicted using PicTar and TargetScan. Among the differentially expressed miRNAs, two miRNAs were selected, miRNA-196b-5p and miRNA-33a-3p, and the target gene of these miRNAs was predicted to be COSMC (data not shown). The present results suggested that there were significant differences miRNA-196b and miRNA-33a expression levels between the $\operatorname{IgAN}$ and control groups $\left(\mathrm{P}=4.73 \times 10^{-7}\right.$ and $\mathrm{P}=0.001$, respectively; data not shown). In addition, as there was a greater difference in the miRNA-196b expression levels compared with the miRNA-33a expression levels between the two groups, miRNA-196b was selected for further analysis in this study.

miRNA-196b expression levels between the IgAN and control groups. B lymphocytes were isolated from the peripheral blood samples of 15 pediatric patients with $\operatorname{IgAN}$ and 15 controls (healthy and other renal diseases). The expression levels of

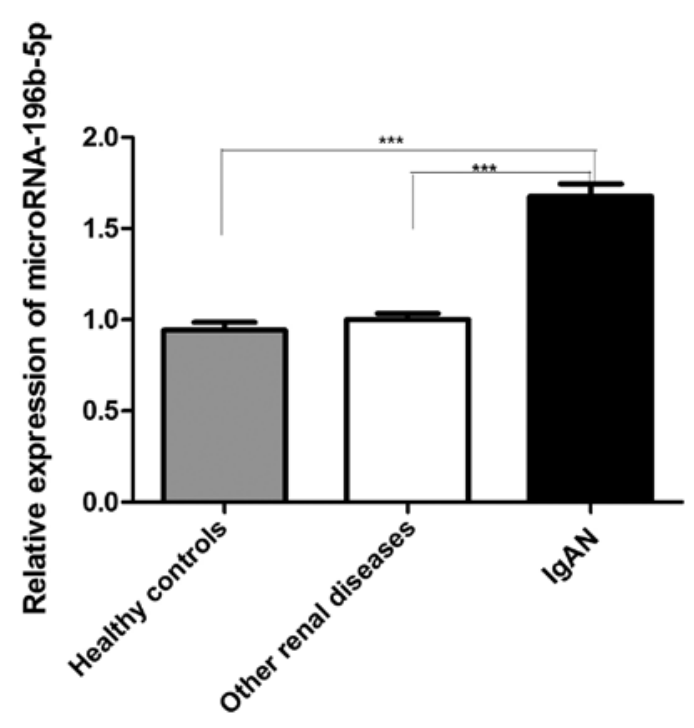

Figure 1. miRNA-196b expression levels in the $\operatorname{IgAN}$, other renal disease and healthy control groups. miRNA-196b expression levels in the IgAN group was significantly increased compared with the control group and the group of patients with other renal diseases. ${ }^{* * * *} \mathrm{P}<0.0001$. miRNA, microRNA; IgAN, immunoglobulin A nephropathy. Error bars represent SD.

miRNA-196b-5p in the IgAN group were significantly higher compared with the healthy control group and the other renal diseases group $(\mathrm{P}<0.0001$; Fig. 1).

Cellular studies. The transfection of miRNA-196b was demonstrated to be successful as significantly increased expression levels of miRNA-196b were observed in the miRNA-196b group $(\mathrm{P}<0.05$; Fig. 2A). Compared with the control and $\mathrm{NC}$ group, COSMC expression levels were decreased in the miRNA-196b group after the addition of the miRNA-196b mimic (Fig. 2B). 


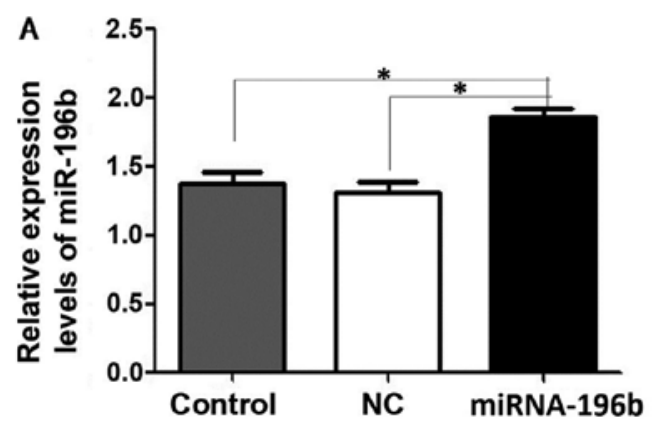

B

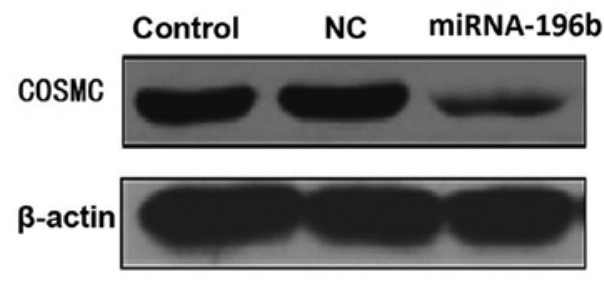

Figure 2. Western blot analysis of COSMC expression levels following the addition of the miRNA-196b mimic. (A) Transfection efficiency of miRNA-196b. Reverse transcription-quantitative PCR was used to analyze the expression levels of miRNA-196b in the control, NC and miRNA-196b-transfected B lymphocytes. (B) Western blot analysis of COSMC expression level after the addition of miRNA-196b mimic. Compared with the control and other renal disease groups, COSMC expression levels decreased after the addition of the miRNA-196b mimic. ${ }^{*} \mathrm{P}<0.05$. COSMC, core1 $\beta 3$-galactosyltransferase-specific molecular chaperone; miRNA, microRNA; IgAN, immunoglobulin A nephropathy.

Gd-IgAl expression levels. In our previous study, compared with the healthy control group $(n=13)$ and other renal disease group ( $\mathrm{n}=11)$, Gd-IgA1 expression levels in the supernatants of B lymphocytes isolated from peripheral blood samples, were significantly increased in the IgAN group (11). In the present study, B lymphocytes from the IgAN group transfected with miRNA-196b mimic $(n=26)$ exhibited significantly increased Gd-IgA1 expression levels compared with the group with no intervention $\left(\mathrm{P}=4.56 \times 10^{-6}\right.$; Fig. 3).

Correlations between miRNA-196b expression levels and clinical data of patients. The results of the present study suggested that there were no significant differences between the IgAN with grade I-II renal damage and the IgAN with grade III-V renal damage groups in terms of miRNA-196b expression levels $(\mathrm{P}=0.609$; Table III). Correlation analysis results suggested that there were no correlations between miRNA-196b expression levels and CD3, CD4 and CD8 expression levels, serum BUN, sCR and GFR levels $(\mathrm{P}=0.186$, $0.875,0.814,0.215,0.117$ and 0.250 , respectively). In addition, there was no significant correlation between miRNA-196b expression and urine red blood cell levels. However, there was a significant correlation between miRNA-196b expression levels and the $24 \mathrm{~h}$ urine total protein level $(\mathrm{P}=0.021$; Table III).

\section{Discussion}

miRNAs are highly conserved non-coding small single-stranded RNAs, $\sim 22$ nucleotides in length, that play crucial roles in gene expression and regulation (17). Different types of miRNAs regulate different target genes (18). In the human genome, $60-70 \%$ of protein-coding genes are regulated by miRNAs and miRNAs have been shown to be associated with various important physiological and pathological processes, such as development, organogenesis, apoptosis, cell proliferation and tumorigenesis (19-22). Previous studies have reported that miRNAs may be involved in the pathophysiology of numerous renal diseases (23-26). Serino et al (13) reported that in patients with IgAN, miRNA-148b expression was downregulated in peripheral blood mononuclear cells and C1ß3Gal-T-1 expression level was decreased compared with normal healthy controls. Moreover, miRNA-148b expression

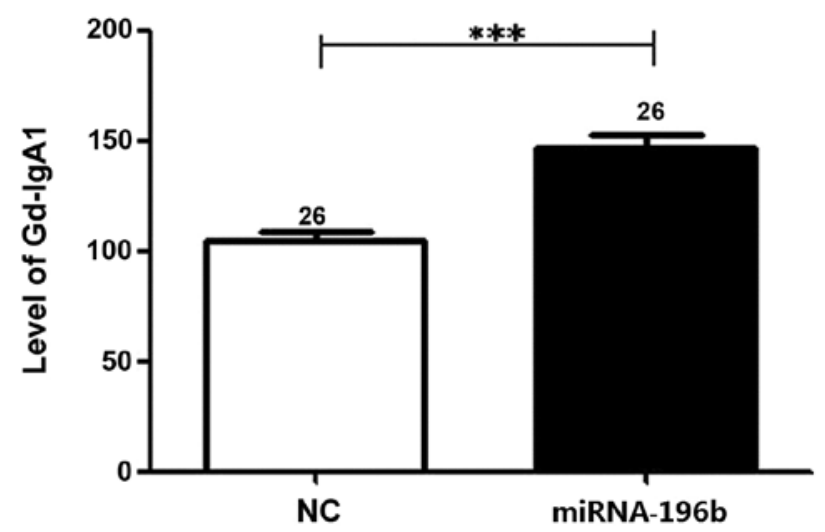

Figure 3. Gd-IgA1 expression levels after the addition of miRNA-196b. In the IgAN group, Gd-IgA1 expression level significantly increased after the addition of miRNA-196b mimic to the supernatant of B lymphocytes compared with the control group with no intervention. ${ }^{* * *} \mathrm{P}<0.001$. Gd-IgA1, galactose-deficient IgA1; IgAN, immunoglobulin A nephropathy; miRNA, microRNA; NC, negative control.

was negatively correlated with C1 $133 \mathrm{Gal}-\mathrm{T}-1$ expression in the same patient cohort (13). These studies suggested that miRNA-148b overexpression in patients with IgAN may inhibit C1 $133 \mathrm{Gal}-\mathrm{T}-1$ expression, thereby leading to aberrant glycosylation of IgA1.

Gd-IgA1 can easily aggregate and form immune complexes that are not cleared as efficiently, which leads to mesangial deposition, cell proliferation and expansion of the extracellular matrix $(2,13)$; this subsequently results in inflammation and glomerular injury (27). However, it remains unknown whether the function of COSMC, the key chaperone of C $1 \beta 3 \mathrm{Gal}-\mathrm{T}$, is regulated by miRNAs (13). Therefore, in the present study, the global expression profile of peripheral blood mononuclear cells obtained from pediatric patients with IgAN was analyzed. Bioinformatics analysis identified 205 miRNAs that were differentially regulated in this patient cohort and suggested that miRNAs may be involved in the pathogenesis of IgAN. In addition, COSMC was identified as one of the target genes of miRNA-196b and miRNA-33a, which were among the 205 detected miRNAs. The present results suggested that there were significant differences miRNA-196b and miRNA-33a expression levels between the IgAN and control groups. As there was a greater difference in terms of miRNA-196b 
expression levels compared with miRNA-33a expression levels between the two groups, miRNA-196b was selected for further analysis. Future studies will investigate miRNA-33a in depth.

The present study demonstrated that miRNA-196b expression increased in human peripheral blood B lymphocytes from the IgAN group compared with the healthy control and other renal disease groups, thus indicating that the abnormal expression was specific to the IgAN group. The other renal disease control group included patients with hereditary nephritis and membranous nephropathy. As the pathogenesis of purpura nephritis is similar to that of $\operatorname{IgAN}(28)$, pediatric patients with purpura nephritis were not included in the control groups. Moreover, future studies will investigate miRNA-196b expression levels between pediatric patients with $\operatorname{IgAN}$ and patients with purpura nephritis.

In addition, the present results suggested that there were no correlations between miRNA-196b expression levels, and CD3, CD4, CD8, BUN, Cr or urine red blood cell levels. Moreover, there were no correlations between miRNA-196b expression levels, and GFR or renal pathology, while there was a significant correlation between miRNA-196b expression levels and $24 \mathrm{~h}$ urine total protein. As $24 \mathrm{~h}$ urine total protein is one of the independent factors of disease severity and prognosis (1), miRNA-196b expression levels may be used as a non-invasive diagnostic marker of IgAN progression. However, due to the limited sample size of the present study, no significant association was identified between miRNA-196b expression level and renal pathological grading. Therefore, future studies with larger sample numbers are underway to investigate the possible correlations between miRNA-196b expression levels and renal pathology with new grading standards.

To investigate whether miRNA-196b can modulate the target gene COSMC expression levels, B lymphocytes of pediatric patients with IgAN were transfected with miRNA-196b mimics. The present results suggested that COSMC expression levels significantly decreased and Gd-IgA1 expression levels increased after miRNA-196b transfection. Therefore, it is hypothesized that increased miRNA-196b expression levels may downregulate COSMC expression levels. Moreover, as the chaperone protein of a key enzyme, reduced COSMC expression levels may affect the normal function of $\mathrm{C} 1 \beta 3 \mathrm{Gal}-\mathrm{T}$, which could result in the formation, accumulation and deposition of deglycosylated IgA1 in serum and the kidneys, subsequently leading to the onset of IgAN.

However, the present study is only a preliminary study, thus the specific mechanism underlying the regulation of COSMC expression by miRNA-196b is not fully understood. Therefore, further cytological experiments are required. In addition, due to the limited number of samples in the present study, further clinical tests with greater numbers of patient samples are needed. In the present study, due to the limited blood samples, it is difficult to perform multiple experimental tests. Future studies will involve animal experiments to investigate the effects in vivo.

Collectively, the present results suggested that miRNA-196b expression levels in B lymphocytes were significantly higher in pediatric patients with IgAN compared with patients with other renal diseases and healthy controls. Therefore, miRNA-196b levels may be related to the clinical characteristics of pediatric patients with IgAN. The transfection of isolated B lymphocytes with miRNA-196b mimics significantly downregulated COSMC expression and increased Gd-IgA1 expression levels.
The present results suggested that miRNA-196b may play crucial roles in the formation of Gd-IgA1 and pathogenesis of IgAN by targeting and regulating COSMC expression. However, the specific underlying mechanisms require further investigation.

\section{Acknowledgements}

Not applicable.

\section{Funding}

The present study was supported by National Natural Science Fund (grant no. 81600551), the Beijing Municipal Science and Technology Commission Major Research Project (grant no. D181100000118006), the Capital Health Research and Development of Special Grant (grant no. 2016-2-2094) and the Application of Capital Clinical Characteristics Program of Beijing Municipal Science and Technology Commission (grant no. Z161100000516106).

\section{Availability of data and materials}

The datasets used and/or analyzed during the current study are available from the corresponding author on reasonable request.

\section{Authors' contributions}

QS and XL analyzed and interpreted the data and were major contributors in the writing of the manuscript. JL, HZ, NZ and YL performed the clinical and laboratory experiments. All authors read and approved the final manuscript.

\section{Ethics approval and consent to participate}

All procedures performed in studies involving human participants were approved by Medical Ethics Committee of Beijing Children's Hospital, Capital Medical University (IRB approval no. 2014-12). Informed consent was obtained from the parents of all participants prior to the study.

\section{Patient consent for publication}

Not applicable.

\section{Competing interests}

The authors declare that they have no competing interest.

\section{References}

1. Seikrit C, Rauen T and Floege J: Immunoglobulin A nephropathy. Internist (Berl) 60: 432-439, 2019.

2. Ebefors K, Liu P, Lassén E, Elvin J, Candemark E, Levan K, Haraldsson B and Nyström J: Mesangial cells from patients with IgA nephropathy have increased susceptibility to galactose-deficient IgA1. BMC Nephrol 17: 40, 2016.

3. Sanders JT, Hastings MC, Moldoveanu Z, Novak J, Julian BA, Bursac Z and Wyatt RJ: Serial galactose-deficient IgA1 levels in children with IgA nephropathy and healthy controls. Int J Nephrol 2017: 8210641, 2017. 
4. Coppo R: Biomarkers and targeted new therapies for $\operatorname{Ig}$ A nephropathy. Pediatr Nephrol 32: 725-731, 2017.

5. Kim MJ, Schaub S, Molyneux K, Koller MT, Stampf S and Barratt J: Effect of immunosuppressive drugs on the changes of serum galactose-deficient IgA1 in patients with IgA nephropathy. PLoS One 11: e0166830, 2016.

6. Lai KN, Tang SC, Schena FP, Novak J, Tomino Y, Fogo AB and Glassock RJ: IgA nephropathy. Nat Rev Dis Primers 2: 16001, 2016

7. Shin DH, Lim BJ, Han IM, Han SG, Kwon YE, Park KS, Lee MJ, Oh HJ, Park JT, Han SH, et al: Glomerular IgG deposition predicts renal outcome in patients with IgA nephropathy. Mod Pathol 29: 743-752, 2016.

8. Ju T and Cummings RD: A unique molecular chaperone Cosmc required for activity of the mammalian core 1 beta 3-galactosyltransferase. Proc Natl Acad Sci USA 99: 16613-16618, 2002.

9. Suzuki H, Yasutake J, Makita Y, Tanbo Y, Yamasaki K, Sofue T, Kano T and Suzuki Y: IgA nephropathy and IgA vasculitis with nephritis have a shared feature involving galactose-deficient IgA1-oriented pathogenesis. Kidney Int 93: 700-705, 2018.

10. Placzek WJ, Yanagawa H, Makita Y, Renfrow MB, Julian BA Rizk DV, Suzuki Y, Novak J and Suzuki H: Serum galactose-deficient-IgA1 and IgG autoantibodies correlate in patients with IgA nephropathy. PLoS One 13: e0190967, 2018.

11. Sun Q, Zhang J, Zhou N, Liu X and Shen Y: DNA methylation in Cosmc promoter region and aberrantly glycosylated IgA1 associated with pediatric IgA nephropathy. PLoS One 10: e0112305, 2015.

12. Serino G, Pesce F, Sallustio F, De Palma G, Cox SN, Curci C, Zaza G, Lai KN, Leung JC, Tang SC, et al: In a retrospective international study, circulating miR-148b and let-7b were found to be serum markers for detecting primary $\operatorname{IgA}$ nephropathy. Kidney Int 89: 683-692, 2016.

13. Serino G, Sallustio F, Cox SN, Pesce F and Schena FP: Abnormal miR-148b expression promotes aberrant glycosylation of IgA1 in IgA nephropathy. J Am Soc Nephrol 23: 814-824, 2012.

14. Livak KJ and Schmittgen TD: Analysis of relative gene expression data using real-time quantitative PCR and the 2(-Delta Delta C(T)) method. Methods 25: 402-408, 2001.

15. Schwartz GJ, Muñoz A, Schneider MF, Mak RH, Kaskel F, Warady BA and Furth SL: New equations to estimate GFR in children with CKD. J Am Soc Nephrol 20: 629-637, 2009.
16. Lee HS, Lee MS, Lee SM, Lee SY, Lee ES, Lee EY, Park SY, Han JS, Kim S and Lee JS: Histological grading of IgA nephropathy predicting renal outcome: Revisiting $\mathrm{H}$. S. Lee's glomerular grading system. Nephrol Dial Transplant 20: 342-348, 2005.

17. Runtsch MC, Hu R, Alexander M, Wallace J, Kagele D, Petersen C, Valentine JF, Welker NC, Bronner MP, Chen X, et al: MicroRNA-146a constrains multiple parameters of intestinal immunity and increases susceptibility to DSS colitis. Oncotarget 6: 28556-28572, 2015.

18. Chandrasekaran K, Karolina DS, Sepramaniam S, Armugam A Wintour EM, Bertram JF and Jeyaseelan K: Role of microRNAs in kidney homeostasis and disease. Kidney Int 81: 617-627, 2012.

19. Wang G, Kwan BC, Lai FM, Chow KM, Li PK and Szeto CC: Elevated levels of miR-146a and miR-155 in kidney biopsy and urine from patients with $\operatorname{Ig}$ A nephropathy. Dis Markers 30: 171-179, 2011.

20. Pauley KM, Cha S and Chan EK: MicroRNA in autoimmunity and autoimmune diseases. J Autoimmun 32: 189-194, 2009.

21. Bi Y, Liu G and Yang R: MicroRNAs: Novel regulators during the immune response. J Cell Physiol 218: 467-472, 2009.

22. Pauley KM and Chan EK: MicroRNAs and their emerging roles in immunology. Ann N Y Acad Sci 1143: 226-239, 2008.

23. Denby L and Baker AH: Targeting non-coding RNA for the therapy of renal disease. Curr Opin Pharmacol 27: 70-77, 2016.

24. Lai JY, Luo J, O'Connor C, Jing X, Nair V, Ju W, Randolph A, Ben-Dov IZ, Matar RN, Briskin D, et al: MicroRNA-21 in glomerular injury. J Am Soc Nephrol 26: 805-816, 2015.

25. Serino G, Sallustio F, Curci C, Cox SN, Pesce F, De Palma G and Schena FP: Role of let-7b in the regulation of $\mathrm{N}$-acetylgalactosaminyltransferase 2 in IgA nephropathy. Nephrol Dial Transplant 30: 1132-1139, 2015.

26. Denby L, Ramdas V, Lu R, Conway BR, Grant JS, Dickinson B, Aurora AB, McClure JD, Kipgen D, Delles C, et al: MicroRNA-214 antagonism protects against renal fibrosis. J Am Soc Nephrol 25: 65-80, 2014.

27. Yeo SC, Cheung CK and Barratt J: New insights into the pathogenesis of IgA nephropathy. Pediatr Nephrol 33: 763-777, 2018.

28. Mao S, Xuan X, Sha Y, Zhao S, Zhu C, Zhang A and Huang S: Clinico-pathological association of Henoch-Schoenlein purpura nephritis and IgA nephropathy in children. Int J Clin Exp Pathol 8: 2334-2342, 2015. 